\title{
THE LACK OF STRICT CONVEXITY AND THE VALIDITY OF THE COMPARISON PRINCIPLE FOR A SIMPLE CLASS OF MINIMIZERS (REVISED VERSION)
}

\author{
CARLO MARICONDA
}

AbstRact. Let $w^{*}(x)=a \cdot x+b$ be an affine function in $\mathbb{R}^{N}, \Omega \subset \mathbb{R}^{N}$, $L: \mathbb{R}^{N} \rightarrow \mathbb{R}$ be convex and $w$ be a local minimizer of

$$
I(v)=\int_{\Omega} L(\nabla v(x)) d x
$$

in $W^{1,1}(\Omega, \mathbb{R})$ with $w(x) \leq w^{*}(x)$ on $\partial \Omega$ in the trace sense. Then $w^{*}$ satisfies the Comparison Principle from above, i.e. $w(x) \leq w^{*}(x)$ a.e. on $\Omega$ if and only if $(a, L(a))$ does not belong to the relative interior of a $N$-dimensional face of the epigraph of $L$. As a consequence, if $F$ is the projection of a bounded face of the epigraph of $L$, the local minimizer of $I w^{*}(x)=\max \left\{\xi \cdot\left(x-x^{0}\right): \xi \in F\right\}$ satisfies the Comparison Principle from above if and only if $\operatorname{dim} F \leq N-1$ or $x^{0} \notin \Omega$.

\section{INTRODUCTION}

We consider the integral functional

$$
I(v)=\int_{\Omega} L(\nabla v(x)) d x
$$

where $L: \mathbb{R}^{N} \rightarrow \mathbb{R} \cup\{+\infty\}$ is convex, lower semicontinuous and $L(\xi) \geq \alpha|\xi|+\beta$, for some $\alpha>0, \beta \in \mathbb{R}$.

A local minimizer of $I$ is a function $w^{*}$ in the Sobolev space $W^{1,1}(\Omega)$ such that $L\left(\nabla w^{*}(x)\right) \in L^{1}(\Omega)$ and $I(u) \geq I(v)$ for every $v$ with $v-w^{*} \in W_{0}^{1,1}(\Omega)$. We say that a local minimizer $w^{*}$ of $I$ satisfies the Comparison Principle (C.P.) from above (resp. from below) if, whenever $w$ is any other local minimizer of $I$, the inequality $w \leq w^{*}$ (resp. $w \geq w^{*}$ ) on the boundary $\Gamma$ of $\Omega$ in the trace sense remains valid a.e. on $\Omega$; the function $w^{*}$ is said to satisfy the C.P. if it satisfies the C.P. both from below and from above.

The Comparison Principles for minimizers of $I$ are a fundamental tool in many recent regularity results with very mild assumptions on the lagrangian: for instance the Lipschitz regularity result of [3], the local Lipschitz regularity of [5], the continuity result of [1] all make use of a Comparison Principle between two functions, one of which is affine.

It is well known that, whenever $L$ is strictly convex, the affine functions whose gradient belongsto the domain of $L$ are local minimizers of $I$ that satisfy the Comparison Principle (see, for instance, [10] for a Comparison Principle among Lipschitz functions, or [3] in the Sobolev setting). However, as it is pointed out in [4], affine

Date: March 12, 2010.

1991 Mathematics Subject Classification. 49K20.

Key words and phrases. Comparison Principle, strict convexity, nonsmooth.

I wish to thank Giulia Treu for the discussions we had during the preparation of this paper and Arrigo Cellina for his critical comments of the first version of the manuscript. 
functions may no more satisfy the Comparison Principle if $L$ is not strictly convex. As an example, if

$$
L(\xi)= \begin{cases}0, & \text { if } \xi \in[-1,1] \\ \left(\xi^{2}-1\right)^{2} & \text { otherwise }\end{cases}
$$

and $\Omega=$ ] $-1,1\left[\right.$ then $w_{1}(x)=1-|x|$ and $w_{2}(x)=\frac{1}{2}(x+1)$ are local minimizers of $I$ satisfying $w_{1} \leq w_{2}$ on $\Gamma=\partial \Omega$; however none of the inequalities $w_{1} \leq w_{2}$ or $w_{2} \leq w_{1}$ holds a.e. on $\Omega$.

It is then natural to ask, in the case where $L$ is not strictly convex, whether there still are some affine functions that do satisfy the Comparison Principle. We show here that the affine function $a \cdot x+b$ satisfies the C.P. if and only if $(a, L(a))$ does not belong to the relative interior of a $N$ - dimensional face of the epigraph of $L$. Both the sufficiency and the necessity of the condition follow from some more general results that have an interest in themselves and that may be applied to wider classes of local minimizers. In particular the sufficiency follows from a Comparison Principle that is directly inspired by those formulated in [3], [4], [13], [12] and that is more suitable to deal in the case where the lagrangian $L$ has some $N$ - dimensional faces. A Comparison result involving a local minimizer and a convex function follows. The necessity would also follow easily from the fact that, if $(a, L(a))$ belongs to a face of the epigraph of $L$ of dimension $N$, then by [2] the problem of minimizing $I$ with $a \cdot x+b$ as a boundary datum has more than a solution.

The lack of the validity, in general, of the C.P. for affine functions when $L$ is not strictly convex induced Cellina to introduce a new class of local minimizers of $I$ in [4]. More precisely, let $F$ be the projection of a bounded face of the epigraph of $L, x^{0} \in \mathbb{R}^{N}$ and consider the function $h_{F, x^{0}}^{+}(x)=\max \left\{\xi \cdot\left(x-x^{0}\right): \xi \in F\right\}$; it is worth noticing that in the case where $L$ is strictly convex, $F$ is reduced to a point and $h_{F, x^{0}}^{+}$is nothing else than an affine function. It was first proven in [4], under the assumption that $\Omega$ be convex and $x^{0} \notin \Omega$, later in [14] by just assuming that $x_{0}$ is a limit of points that do not belong to the closure of $\Omega$, that $h_{F, x^{0}}^{+}$ satisfies the Comparison Principle from above. As it is pointed out in [4] the result may be false if $x^{0} \in \Omega$ : this can be seen again if $L$ is defined by (1): by taking $F=[-1,1]$ and $x^{0}=0$ we obtain that $h_{F, 0}^{+}=|x|$; however $w(x)=\frac{1}{2} x+\frac{1}{2}$ is a local minimizer of $I$ on ] $-1,1\left[\right.$ with $w \leq h_{F, 0}^{+}$on $\partial \Omega$ but $w>h_{F, 0}^{+}$on a non negligible set. As an application of the characterization of the affine functions that satisfy the Comparison Principle we deduce that the function $h_{F, x^{0}}^{+}$may satisfy the C.P. even if $x^{0} \in \Omega$; more precisely $h_{F, x^{0}}^{+}$satisfies the Comparison Principle from above if and only if either the affine dimension of $F$ is at most equal to $N-1$ or if $x^{0} \notin \Omega$; moreover if $x^{0} \notin \Omega$ it turns out that $h$ satisfies the Comparison Principle also from below.

\section{ASSUMPTIONS AND NOTATION}

The function $L: \mathbb{R}^{N} \rightarrow \mathbb{R} \cup\{+\infty\}$ is convex, lower semicontinuous and moreover $L(\xi) \geq \alpha|\xi|+\beta$ for some $\alpha \geq 0, \beta \in \mathbb{R}$. The domain of $L$ is

$$
\operatorname{Dom}(L)=\left\{\xi \in \mathbb{R}^{N}: L(\xi)<+\infty\right\}
$$

and the subdifferential of $L$ at $\xi^{0} \in \operatorname{Dom}(L)$ is the set

$$
\partial L\left(\xi^{0}\right)=\left\{p \in \mathbb{R}^{N}: \forall \xi \in \mathbb{R}^{N} \quad L(\xi)-L\left(\xi^{0}\right) \geq p \cdot\left(\xi-\xi^{0}\right)\right\}
$$

where "." is the standard scalar product in $\mathbb{R}^{N}$; we recall that $\partial L(x)$ is non empty and bounded at every point $x$ that is interior to $\operatorname{Dom}(L)$. The polar of $L$ is

$$
L^{*}(p)=\sup \left\{p \cdot \xi-L(\xi): \xi \in \mathbb{R}^{N}\right\} .
$$


$\Omega$ is an open and bounded subset of $\mathbb{R}^{N}$, its boundary is $\Gamma$. Geometrically, for $p$ in the domain of $L^{*}, \partial L^{*}(p)$ is the projection onto $\mathbb{R}^{N}$ of the exposed face of the epigraph of $L$ whose normal vector is $(p,-1)$; moreover $x \in \partial L^{*}(p)$ if and only if $p \in \partial L(x)$.

By $\mathcal{C}_{c}^{\infty}(\Omega)$ we denote the functions of class $\mathcal{C}^{\infty}$ in $\Omega$ with compact support. If $S \in L_{\text {loc }}^{1}\left(\Omega, \mathbb{R}^{N}\right)$ we write that $\operatorname{div} S=0$ if the divergence of $S$ is equal to zero in the distributional sense, i.e.

$$
\forall \varphi \in \mathcal{C}_{c}^{\infty}(\Omega) \quad \int_{\Omega} S(x) \cdot \nabla \varphi(x) d x=0 .
$$

If $C$ is a convex subset of $\mathbb{R}^{N}$, its affine dimension is $\operatorname{dim} C$. The interior of $A \subseteq \mathbb{R}^{N}$ is int $A$, its boundary is bd $A$.

The functional $I$ is defined on $W^{1,1}(\Omega)$ by

$$
I(v)=\int_{\Omega} L(\nabla v(x)) d x
$$

\section{A Comparison Principle}

Let $u, v \in W^{1,1}(\Omega)$; in this setting by $u \leq v$ on $\Gamma$ we mean that the positive part $(u-v)^{+}$of $u-v$ belongs to $W_{0}^{1,1}(\Omega)$.

Definition 1. Let $w^{*}$ be a local minimizer of $I$. We say that $w^{*}$ satisfies the Comparison Principle from above (resp. from below) if for every local minimizer $w$ of $I$ in $W^{1,1}(\Omega)$ the inequality $w \leq w^{*}$ (resp. $\left.w^{*} \leq w\right)$ on $\Gamma$ implies $w \leq w^{*}$ (resp. $\left.w^{*} \leq w\right)$ a.e. on $\Omega$. Finally, we say that $w^{*}$ fulfills the Comparison Principle (or C.P.) if $w^{*}$ fulfills both the C.P. from above and from below.

For $\alpha>0$ and $u \in \mathbb{R}^{N}$ with $|u|=1$ let us denote by $C(u, \alpha)$ the cone

$$
C(u, \alpha)=\left\{y \in \mathbb{R}^{N}: y \cdot u \geq \alpha|y|\right\}
$$

The condition stated hereafter is directly inspired to the cone property introduced in [11]; its formulation here is slightly less restrictive than the original one.

Definition 2 (The cone property revisited). Let $T: \Omega \rightarrow \mathbb{R}^{N} \backslash\{0\}$ be Lipschitz; for $z \in \Omega$ denote by $x_{z}:\left[0, t(z)\left[\rightarrow \Omega\right.\right.$ the maximal solution on the right of $x^{\prime}=$ $T(x) \quad x(0)=z$. We say that the vector field $T$ satisfies the cone property if for every $\bar{x} \in \Omega$ there exist a ball $B(\bar{x}, r) \subset \Omega$ centered in $\bar{x}$ and of radius $r$, a vector $u$ in $\mathbb{R}^{N}$ and $\alpha>0$ such that

$$
\forall z \in B(\bar{x}, r) \quad \forall t \in\left[0, t(z)\left[\quad T\left(x_{z}(t)\right) \in C(u, \alpha)\right.\right.
$$

Remark 1. It is easy to show that, whenever $T$ is a vector field that satisfies the cone property, the trajectories of the system $x^{\prime}=T(x)$ exit from $\Omega$ in a finite time, i.e. $t(z)<+\infty$ and $x(t(z)) \doteq \lim _{t \rightarrow t^{-}(z)} x_{z}(t) \in \Gamma$.

Example 1. Let $x^{0} \notin \Omega$ and set $T(x)=x-x^{0}$. Then $T$ and $-T$ satisfy the cone property. Indeed fix $\bar{x} \in \Omega$ and let $r<\left|\bar{x}-x^{0}\right|$. Then for every $z$ in $B(\bar{x}, r)$ we have

$$
\left(z-x^{0}\right) \cdot \frac{\bar{x}-x^{0}}{\left|\bar{x}-x^{0}\right|}=\left((z-\bar{x})+\left(\bar{x}-x^{0}\right)\right) \cdot \frac{\bar{x}-x^{0}}{\left|\bar{x}-x^{0}\right|} \geq\left|\bar{x}-x^{0}\right|-r
$$

so that, for $z \in B(\bar{x}, r)$,

$$
\frac{z-x^{0}}{\left|z-x^{0}\right|} \cdot u \geq \alpha \text { where } u=\frac{\bar{x}-x^{0}}{\left|\bar{x}-x^{0}\right|}, \quad \alpha=\frac{\left|\bar{x}-x^{0}\right|-r}{\left|\bar{x}-x^{0}\right|+r}
$$


Now let $z \in B(x, r)$; the solution to $x^{\prime}=T(x)$ with the initial condition $x(0)=z$ is given by $x_{z}(t)=z e^{t}+x^{0}\left(1-e^{t}\right)(t>0)$ so that

$$
\frac{x_{z}(t)-x^{0}}{\left|x_{z}(t)-x^{0}\right|} \cdot u=\frac{z-x^{0}}{\left|z-x^{0}\right|} \geq \alpha
$$

or, equivalently,

$$
\forall z \in B(x, r) \quad T\left(x_{z}(t)\right) \in C(u, \alpha) .
$$

The same arguments show that $-T$ satisfies the cone property.

Example 2. A non zero Lipschitz vector field $T$ satisfies the cone property if, for instance, there exist $u \in \mathbb{R}^{N},|u|=1$ and $\alpha>0$ such that

$$
\forall x \in \Omega \quad T(x) \in C(u, \alpha)
$$

This is actually the original definition of the cone property that was given in [11], more restrictive than the one that is given here. For instance, the vector field $T(x)=x-x^{0}$ studied in Example 1 satisfies the cone property whenever $x^{0} \notin \Omega$ but it may not fulfill (3) if $x^{0}$ lies in the relative interior of a flat part of the boundary of $\Omega$. This particular vector field will be used in the proof of Theorem 3 .

It is shown in [11, Theorem 3.2] that, if $T$ satisfies condition (2) then the functions of $W_{0}^{1,1}(\Omega)$ admit a representative that is absolutely continuous along a.e. trajectory of $x^{\prime}=T(x)$; the original proof of this result does still hold true if $T$ satisfies the cone property redefined here.

Definition 3 (The order relation $\geq_{u}$ ). For any vector $u$ of $\mathbb{R}^{N}$ let us denote by $\geq_{u}$ the order relation induced by $u$ defined by

$$
\forall x, y \in \mathbb{R}^{N} \quad x \geq_{u} y \Longleftrightarrow(x-y) \cdot u \geq 0 ;
$$

if $A$ is a subset of $\mathbb{R}^{N}$ we write $A \geq_{u} y$ (resp. $x \geq_{u} A$ ) if $\xi \geq_{u} y$ (resp. $x \geq_{u} \xi$ ) for every $\xi \in A$.

Theorem 1 (A general Comparison Principle). Let $w^{*}$ be a local minimizer of I that satisfies the Euler equation, i.e. there is $S \in L_{\mathrm{loc}}^{1}\left(\Omega ; \mathbb{R}^{N}\right)$ such that

$$
\operatorname{div} S=0 \quad S(x) \in \partial L\left(\nabla w^{*}(x)\right) \text { a.e. }
$$

Let $T: \Omega \rightarrow \mathbb{R}^{N}$ be Lipschitz and such that either

i) $\operatorname{Dom}(L)$ is bounded and the trajectories from $\Omega$ of $x^{\prime}=T(x)$ leave $\Gamma$ in a finite time, or

ii) $S$ is bounded and $T$ satisfies the cone property.

Moreover, assume that

$$
\partial L^{*}(S(x)) \geq_{T(x)} \nabla w^{*}(x) \text { a.e. }
$$

Then $w^{*}$ satisfies the Comparison Principle from above. If, instead of (5),

$$
\nabla w^{*}(x) \geq_{T(x)} \partial L^{*}(S(x)) \text { a.e. }
$$

then $w^{*}$ satisfies the Comparison Principle from below.

Remark 2. This version of the Comparison Principle generalizes, in the case where $L$ depends only on the gradient, the versions of the C.P. that appeared in the papers [3], [4], [13], [12]. Actually, in [3], [4] and [13], the C.P. was referred to a particular minimizer; in [12], the more restrictive condition (3) was assumed instead of the cone property and conditions (5)-(6) were replaced by the less general condition that the field $T$ be orthogonal to $\partial L^{*}(S(x))$. 
Remark 3. The assumption, in Theorem 1, that the minimizer $w^{*}$ satisfies the Euler equation is fulfilled if the minimizer is known to be locally Lipschitz or if $L$ is locally Lipschitz and its growth is less than exponential [6, Theorem 4.6.1]. A recent result [9] asserts that the Euler equation does hold if $L$ is smooth and the boundary datum is locally bounded. It is not known however whether the Euler equation is satisfied in the general case.

The arguments of the proof of Theorem 1 are similar to those used in [12], [14]; we just stress here how the less restrictive conditions (5)-(6) are used to obtain the conclusion.

Proof. Proof of Theorem 1 Let $w$ be a local minimizer of $I$ satisfying $w \leq w^{*}$ on $\Gamma$. Assume that $w>w^{*}$ on a subset $E$ of $\Omega$. Set $\eta=\left(w-w^{*}\right)^{+} \doteq \max \left\{w-w^{*}, 0\right\}$, so that $E=\{x \in \Omega: \eta(x)>0\}$; our assumption implies that $\eta \in W_{0}^{1,1}(\Omega)$, actually $\eta \in W_{0}^{1, \infty}(\Omega)$ if $\operatorname{Dom}(L)$ is bounded: in this case both $\nabla w$ and $\nabla w^{*}$ are bounded. Since $\min \left\{w, w^{*}\right\}=w$ and $\max \left\{w, w^{*}\right\}=w^{*}$ on $\Gamma$ then $I\left(\min \left\{w, w^{*}\right\}\right) \geq I(w)$ and $I\left(\max \left\{w, w^{*}\right\}\right) \geq I\left(w^{*}\right)$ and thus

$$
\int_{E} L\left(\nabla w^{*}(x)\right) d x=\int_{E} L(\nabla w(x)) d x
$$

Moreover the validity of the Euler equation implies that

$$
\int_{E} S(x) \cdot \nabla \eta(x) d x=\int_{\Omega} S(x) \cdot \nabla \eta(x) d x=0
$$

if either $\eta \in W_{0}^{1, \infty}(\Omega)$ as in i), or if $\eta \in W_{0}^{1,1}(\Omega)$ and $S$ is bounded as in ii). Thus in both cases we have

$$
\int_{\Omega} S(x) \cdot\left(\nabla w(x)-\nabla w^{*}\right)(x) d x=0
$$

so that

$$
\int_{E} L(\nabla w(x)) d x=\int_{E} L\left(\nabla w^{*}(x)\right)+S(x) \cdot\left(\nabla w(x)-\nabla w^{*}\right)(x) d x .
$$

However, since $S(x) \in \partial L\left(\nabla w^{*}(x)\right)$, then

$$
L(\nabla w(x)) \geq L\left(\nabla w^{*}(x)\right)+S(x) \cdot\left(\nabla w(x)-\nabla w^{*}(x)\right) \text { a.e. on } E .
$$

It follows that the latter inequality is actually an equality a.e. on $E$ and thus

$$
\nabla w(x) \in \partial L^{*}(S(x)) \text { a.e. on } E \text {. }
$$

Our assumption (5) then implies that $\nabla w(x) \geq_{T(x)} \nabla w^{*}(x)$, i.e.

$$
T(x) \cdot \nabla w(x) \geq T(x) \cdot \nabla w^{*}(x) \text { a.e. on } E
$$

or, equivalently,

$$
T(x) \cdot \nabla \eta(x) \geq 0 \text { a.e. }
$$

If i) holds then $\eta$ is Lipschitz and vanishes on $\Gamma$ : we fix $z \in E$. If, instead, ii) holds let $B(\bar{x}, r), u, \Pi$ be as in [11, Theorem 3.2]; $\eta$ has a representative $\eta^{*}$ that is absolutely continuous on a.e. trajectory of $x^{\prime}=T(x)$ starting from $\Pi$ and vanishes at the end point of the trajectory in $\Gamma$ : we fix $z \in \Pi \cap B(\bar{x}, r) \cap E$. In any case the map $t \mapsto \eta^{*}\left(x_{z}(t)\right)$ is absolutely continuous on $[0, t(z)[$ and

$$
x_{z}(0)=z, \quad x^{*}=\lim _{t \rightarrow t(z)} x_{z}(t) \in \Gamma, \quad \eta^{*}\left(x^{*}\right)=0 .
$$

For every $t \in[0, t(z)[$ we have

$$
\eta^{*}\left(x_{z}(t)\right)-\eta^{*}(z)=\int_{0}^{t} \nabla \eta^{*}\left(x_{z}(s)\right) \cdot T\left(x_{z}(s)\right) d s \geq 0
$$


so that, passing to the limit for $t \rightarrow t(z)$, we obtain that $0 \geq \eta^{*}(z)$. Therefore $\eta^{*} \leq 0$ a.e. on $E$. However, since $\eta^{*}>0$ a.e. on $E$ it turns out that $E$ is negligible, proving that $w \leq w^{*}$ a.e.. on $\Omega$. If (6) holds instead of (5), the proof of the Comparison Principle from below follows similarly.

The following consequence of Theorem 1 is immediate since $x \geq_{u} y$ if and only if $y \geq(-u) x$.

Corollary 1. Under the above assumptions, assume that both $T$ and $-T$ satisfy the cone property and that just one of the conditions (5) or (6) holds. Then $w^{*}$ satisfies the Comparison Principle both from above and from below.

In the next section we are mainly concerned in the case where the field $S$ involved in Theorem 1 is constant. As an application of Theorem 1 in a case where $S$ is a non constant divergence free field we give here a shorter alternative proof of $[7$, Theorem 2.1].

Example 3. We consider a radial Lagrangean of the form $L(\xi)=\ell(|\xi|)$ where $\ell: \mathbb{R} \rightarrow \mathbb{R} \cup\{+\infty\}$ is convex, even, lower semicontinuous and $\ell(x)=+\infty$ for $x$ out of a bounded interval. Let $z^{*}$ be a measurable selection of $\ell^{*}$. Assuming that $0 \notin \Omega$ it is shown in [7] that the function $w^{*}$ defined by

$$
w^{*}(x)=\int_{1}^{|x|} z^{*}\left(\frac{1}{s^{N-1}}\right) d s
$$

is a local minimum of $I(u)=\int \ell(\nabla u(x)) d x$ in $W^{1,1}(\Omega)(N \geq 3)$ and that, moreover, $S(x) \doteq \frac{x}{|x|^{N}} \in \partial L^{*}\left(\nabla w^{*}(x)\right)$. Since the field $S$ is equal to a constant times the gradient of a harmonic function it turns out obviously that $\operatorname{div} S=0$, so that $S$ fulfills (4) of Theorem 1 . Now, since

$$
\partial L^{*}(p)=\partial \ell^{*}(|p|) \frac{p}{|p|} \text { if } p \neq 0, \quad \partial L^{*}(0)=\partial \ell^{*}(0) B(0,1]
$$

where $B(0,1]$ is the closed unit ball of $\mathbb{R}^{N}$, it turns out that $\partial L^{*}(S(x))$ is reduced to a point unless $1 /|x|^{N-1}$ belongs to the set of values $\lambda>0$ such that $\partial \ell^{*}$ is a non trivial interval, at most a countable set. Therefore $\partial L^{*}(S(x))$ is equal to a point unless $x$ belongs to a countable union of spheres, a negligible set. It follows that any non zero constant field $T$ fulfills (5) and (6); Theorem 1 then implies that $w^{*}$ satisfies the Comparison Principle.

\section{MAIN RESUlts}

The following result will be used in the characterization of the affine functions that satisfy the cone property; it concerns actually a much wider class of local minimizers.

Lemma 1 (A necessary condition). Let $w^{*}$ be a Lipschitz local minimizer of I and assume that $w^{*}$ satisfies the Comparison Principle. Assume moreover that $w^{*} \in W^{2, r}(\Omega)$ for some $r>N$. Then, for a.e. $x,\left(\nabla w^{*}(x), L\left(\nabla w^{*}(x)\right)\right)$ does not belong to the relative interior of a $N$-dimensional face of the epigraph of $L$.

Proof. Assume, by contradiction, that $\left(\nabla w^{*}(x), L\left(\nabla w^{*}(x)\right)\right)$ belongs to the relative interior of a $N$-dimensional face of the epigraph of $L$ on a non negligible set: then there is $p$ in $\mathbb{R}^{N}$ such that $\nabla w^{*}$ belongs to the interior int $\partial L^{*}(p)$ of the convex subdifferential of $L^{*}$ at $p$ on a non negligible set. The regularity assumption on $w^{*}$ implies that

$$
\Omega_{1}=\left\{x \in \Omega: \nabla w_{6}^{*}(x) \in \operatorname{int} \partial L^{*}(p)\right\}
$$


is open. By [8, Theorem 2.10] or [2] there exists a Lipschitz function $u$ on $\Omega_{1}$ such that

$$
\begin{cases}\nabla u(x) \in \operatorname{bd} \partial L^{*}(p) & \text { a.e. in } \Omega_{1} \\ u(x)=w^{*}(x) & \text { on bd } \Omega_{1} .\end{cases}
$$

Let $\widetilde{w}$ be the function defined by

$$
\widetilde{w}(x)= \begin{cases}w^{*}(x) & \text { if } x \notin \Omega_{1} \\ u(x) & \text { if } x \in \Omega_{1}\end{cases}
$$

Then $\widetilde{w}=w^{*}$ on $\Gamma$; moreover $\widetilde{w}$ is a local minimizer of $I$. Indeed

$$
I(\widetilde{w})=\int_{\Omega} L(\nabla \widetilde{w}(x)) d x=\int_{\Omega_{1}} L(\nabla u(x)) d x+\int_{\Omega \backslash \Omega_{1}} L\left(\nabla w^{*}(x)\right) d x
$$

and since, for a.e. $x$ in $\Omega_{1}, p \in \partial L(\nabla u(x)) \cap \partial L\left(\nabla w^{*}(x)\right)$ then

$$
L(\nabla u(x))=L\left(\nabla w^{*}(x)\right)+p \cdot \nabla\left(u-w^{*}\right)(x)
$$

so that, using the fact that $u-w^{*} \in W_{0}^{1,1}(\Omega)$,

$$
\int_{\Omega_{1}} L(\nabla u(x)) d x=\int_{\Omega_{1}} L\left(\nabla w^{*}(x)\right) d x
$$

thus

$$
I(\widetilde{w})=\int_{\Omega_{1}} L\left(\nabla w^{*}(x)\right) d x+\int_{\Omega \backslash \Omega_{1}} L\left(\nabla w^{*}(x)\right) d x=I\left(w^{*}\right)
$$

and therefore $I(\widetilde{w})=I\left(w^{*}\right), w^{*}$ being a local minimizer of $I$. Now $\widetilde{w}=w^{*}$ on $\Gamma$ but $\widetilde{w} \neq w^{*}$ on a non negligible set, otherwise $\nabla w^{*}=\nabla \widetilde{w} \in \operatorname{bd} \partial L^{*}(p)$ a.e. on $\Omega_{1}$, contradicting the assumption that $\nabla w^{*}$ belongs to int $\partial L^{*}(p)$ on a set of strictly positive measure; it follows that $w^{*}$ does not satisfy the Comparison Principle.

Remark 4. The proof of Lemma 1 shows that the conclusion holds true, without assuming that $w^{*} \in W^{2, r}(\Omega)$, if $\nabla w^{*}$ belongs a.e. to a prescribed face of the epigraph of $L$. Indeed in this case there is $p \in \mathbb{R}^{N}$ such that $\nabla w^{*} \in \partial L^{*}(p)$ a.e. on $\Omega$.

Remark 5. The converse of Lemma 1 is not true, in general. Consider for instance the functional mentioned in the introduction where $L$ is defined by (1): in this case $\partial L^{*}(0)=[-1,1]$ and any function $w^{*}$ whose derivative belongs a.e. to $\operatorname{bd} \partial L^{*}(0)=$ $\{-1,1\}$ is a minimizer of $I$; however $w^{*}(x)=1-|x|$ does not satisfy the Comparison Principle.

Theorem 2 (The affine functions that satisfy the C.P.). Let a belong to the interior of $\operatorname{Dom}(L)$ and $b \in \mathbb{R}$. The affine function $w^{*}(x)=a \cdot x+b$ satisfies the Comparison Principle if and only if $(a, L(a))$ does not belong to the relative interior of a $N$ dimensional face of the epigraph of $L$.

Proof. Assume that $(a, L(a))$ does not belong to the relative interior of a $N$ - dimensional face of the epigraph of $L$. Then there is $p$ in $\mathbb{R}^{N}$ such that $a$ belongs to the boundary of $\partial L^{*}(p)$. Let $\nu$ in $\mathbb{R}^{N}$ be such that

$$
\partial L^{*}(p) \subseteq\left\{\xi \in \mathbb{R}^{N}: \nu \cdot(\xi-a) \geq 0\right\}
$$

Then

$$
\nu \cdot a=\min \left\{\nu \cdot \xi: \xi \in \partial L^{*}(p)\right\}
$$

so that the constant field $T$ satisfies (5) with $S(x)=p$. Moreover $T$ and $-T$ satisfy the cone property: Corollary 1 show that $w^{*}$ satisfies the Comparison Principle. Conversely, if $(a, L(a))$ belongs to the relative interior of a $N$-dimensional face of the epigraph of $L$ then $\partial L(a)$ is a singleton $\{p\}$ and $\nabla w^{*}(x)=a$ belongs to the interior to $\partial L^{*}(p)$ for every $x$ : the conclusion that $w^{*}$ does not satisfy the C.P. follows from Lemma 1. Alternatively one could remark that, from [2], if $(a, L(a))$ 
belongs to the relative interior of a $N$-dimensional face of the epigraph of $L$ then the problem of minimizing $I$ with boundary datum equal to $a \cdot x+b$ has no uniqueness implying in particular the failure of the Comparison Principle.

As an application, we formulate a criteria to compare minimizers and convex functions. A similar conclusion, under a different assumption, was obtained in [1], $[15]$.

Proposition 1. Let $\psi$ be a convex on the closure of $\Omega$ and $w$ be a local minimizer of $I$ such that $w \geq \psi$ on $\Gamma$. Assume that for every $x$ in $\Omega$ there is $\zeta \in \partial \psi(x)$ such that $(\zeta, L(\zeta))$ belongs to a face of the epigraph of $L$ dimension less that $N$. Then $w(x) \geq \psi(x)$ a.e..

Proof. Let $x \in \Omega$ and $\zeta \in \partial \psi(x)$ be as in the claim of the proposition. Then

$$
\forall \gamma \in \Gamma \quad w(\gamma) \geq \psi(\gamma) \geq \zeta \cdot(\gamma-x)+\psi(x) \doteq g(\gamma)
$$

Since $(\zeta, L(\zeta))$ does not belong to a $N$-dimensional face of the epigraph of $L$, Theorem 2 yields that $w(y) \geq g(y)$ a.e. on $\Omega$. By taking the integral mean value of both terms of the inequality when $y$ varies on the balls centered in $x$ we obtain, by passing to the limit as the radii of the balls tend to 0 , that $w(x) \geq g(x)=\psi(x)$ if $x$ is a Lebesgue point of $w$.

Definition 4 (The functions $h_{p, x^{0}}^{+}$and $h_{p, x^{0}}^{-}$). Let $p \in \operatorname{Dom}\left(L^{*}\right)$ be such that $\partial L^{*}(p)$ is bounded. For $x^{0} \in \mathbb{R}^{N}$ we set

$$
h_{p, x^{0}}^{+}(x)=\max _{\xi \in \partial L^{*}(p)} \xi \cdot\left(x-x^{0}\right) \quad h_{p, x^{0}}^{-}(x)=\min _{\xi \in \partial L^{*}(p)} \xi \cdot\left(x-x^{0}\right)
$$

Remark 6. The function $h_{p, x^{0}}^{ \pm}$were first defined in [4] and then thoroughly studied in [14]. In the case where $L$ is strictly convex the set $\partial L^{*}(p)$ is reduced to a point: in this case the functions $h_{p, x^{0}}^{ \pm}$are nothing more than affine. The interest in this class of functions is that, under suitable assumptions, they are minimizers that satisfy the Comparison Principle.

We recall here the main properties of $h_{p, x^{0}}^{ \pm}$formulated in [4].

Proposition 2. Let $p \in \operatorname{Dom}\left(L^{*}\right)$ be such that $\partial L^{*}(p)$ is bounded. The functions $h_{p, x^{0}}^{ \pm}$are Lipschitz minimizers of $I$. Moreover $\nabla h_{p, x^{0}}^{ \pm}(x) \in \partial L^{*}(p)$ a.e. and

$$
\begin{aligned}
& \nabla h_{p, x^{0}}^{+}(x) \cdot\left(x-x^{0}\right)=\max \left\{\xi \cdot\left(x-x^{0}\right): \xi \in \partial L^{*}(p)\right\} \text { a.e. } \\
& \nabla h_{p, x^{0}}^{-}(x) \cdot\left(x-x^{0}\right)=\min \left\{\xi \cdot\left(x-x^{0}\right): \xi \in \partial L^{*}(p)\right\} \text { a.e. }
\end{aligned}
$$

It is shown, under the assumption that $\Omega$ is convex and $x^{0} \notin \Omega$ in [4], just assuming that $x^{0}$ is the limit of points out of the closure of $\Omega$ in [14], that $h_{p, x^{0}}^{+}$ (resp. $h_{p, x^{0}}^{+}$) satisfies the C.P. from above (resp. from below). The next result shows that the result holds true even under some less restrictive assumptions that turn out, moreover, to be also necessary.

Theorem 3 (The functions $h_{p, x^{0}}^{+}$that satisfy the C.P.). Let $p \in \operatorname{Dom}\left(L^{*}\right)$ be such that $\partial L^{*}(p)$ is bounded and $x^{0} \in \mathbb{R}^{N}$. The function $h_{p, x^{0}}^{+}$satisfies the Comparison Principle from above if and only if either $\operatorname{dim} \partial L^{*}(p) \leq N-1$ or $x^{0} \notin \Omega$. Moreover, if $x^{0} \notin \Omega$, then $h_{p, x^{0}}^{+}$satisfies the Comparison Principle both from below and from above.

Remark 7. A dual statement holds obviously for the functions $h_{p, x^{0}}^{-}$: it is enough to replace, in the formulation of Theorem 3, the term above with below. 
Proof. Let $w$ be a local minimizer such that $w \leq h_{p, x^{0}}^{+}$on $\partial \Omega$. Assume that $\operatorname{dim} \partial L^{*}(p) \leq N-1$. Then for every $\xi \in \partial L^{*}(p)$ and $x \in \mathbb{R}^{N}$ we have $\xi \cdot\left(x-x^{0}\right) \leq$ $h_{p, x^{0}}^{+}(x) \leq w(x)$ on $\Gamma$. By Theorem 2 the affine function $x \mapsto \xi \cdot\left(x-x^{0}\right)$ satisfies the C.P. and thus $\xi \cdot\left(x-x^{0}\right) \leq h_{p, x^{0}}^{+}(x) \leq w(x)$ a.e. on $\Omega$ so that

$$
h_{p, x^{0}}^{+}(x)=\max _{\xi \in \partial L^{*}(p)} \xi \cdot\left(x-x^{0}\right) \leq w(x) \text { a.e. }
$$

Assume now that $x^{0} \notin \Omega$ and set $T(x)=x^{0}-x$. It follows from (8) that $\nabla h_{p, x^{0}}^{+}(x) \leq_{T(x)} \partial L^{*}(p)$ a.e., thus ensuring the validity of (5). Example 1 shows that $T(x)= \pm\left(x-x^{0}\right)$ are fields that satisfy the cone property: it follows from Theorem 1 and Corollary 1 that $h_{p, x^{0}}^{+}$satisfies the Comparison Principle.

Finally, assume that $x^{0} \in \Omega$; it is not restrictive to suppose that $x^{0}=0$, we set $h=h_{p, 0}^{+}$for an easier notation. If $\operatorname{dim} \partial L^{*}(p)=N$ then $\partial L^{*}(p)$ contains a ball, say of center $a \in \mathbb{R}^{N}$ and radius $r>0$. It follows that

$$
\forall x \in \mathbb{R}^{N} \quad h(x)=\max _{\xi \in \partial L^{*}(p)} \xi \cdot x \geq \max _{u:|u| \leq 1}(a+r u) \cdot x=a \cdot x+r|x|
$$

Then, if $m \doteq \min _{x \in \Gamma}|x|$ and $w(x) \doteq a \cdot x+r m$, by (10) we have $w \leq h$ on $\Gamma$; however since $0 \notin \Gamma$ then $m>0$ and thus $w(0)=r m>h(0)$. Now the affine function $w$ is a local minimizer of $I$ with $w \leq h$ on $\Gamma$ but $w>h$ on a neighborhood of 0: it follows that $h$ does not satisfy the C.P. from above.

Remark 8. Theorem 3 improves [14, Theorem 3.2], itself an extension of [4] stating that the function $h_{p, x^{0}}^{+}\left(\right.$resp. $\left.h_{p, x^{0}}^{+}\right)$satisfy the C.P. from above (resp. from below) if $x^{0}$ is the limit of points out of the closure of $\Omega$. In Theorem 3 this condition on $x^{0}$ is dropped away if $\operatorname{dim} \partial L^{*}(p) \leq N-1$ and is weakened in the case where $\operatorname{dim} \partial L^{*}(p)=N$ since it is assumed here just that $x^{0} \notin \Omega$. Moreover, if $x^{0} \notin \Omega$, the fact that $h_{p, x^{0}}^{ \pm}$satisfy both the C.P. from above and from below is new to the author.

\section{REFERENCES}

[1] P. Bousquet, Boundary continuity of solutions to a basic problem in the calculus of variations, Adv. Calc. Var. 3 (2010) 1-27.

[2] A. Cellina, On minima of a functional of the gradient: sufficient conditions, Nonlinear Anal. 20 (1993), 343-347.

[3] A. Cellina, On the bounded slope condition and the validity of the Euler Lagrange equation, SIAM J. Control Optim. $40(2001 / 2)$ 1270-1279.

[4] A. Cellina, Comparison results and estimates on the gradient without strict convexity, SIAM J. Control Optim. 46 (2007), 738-749.

[5] F.H. Clarke, Continuity of solutions to a basic problem in the calculus of variations, Ann. Sc. Norm. Super. Pisa Cl. Sci. 4 (2005), 511-530.

[6] F.H. Clarke, Optimization and nonsmooth analysis, John Wiley \& Sons Inc., 1983.

[7] A. Cellina and C. Mariconda and G. Treu, Comparison results without strict convexity, Disc. Cont. Din. Syst. Series B 11 (2009), 57-65.

[8] B. Dacorogna and P. Marcellini, Implicit Partial Differential Equations, Progress in Nonlinear Differential Equations and their Applications, Vol. 37, Birkhäuser, 1999.

[9] M. Degiovanni and M. Marzocchi, On the Euler-Lagrange equation for functionals of the calculus of variations without upper growth conditions, SIAM J. Control Optim. 48 (2009), $2857-2870$.

[10] E. Giusti, Direct Methods in the Calculus of Variations, World Scientific, 2003.

[11] C. Mariconda and G. Treu, Absolutely continuous representatives on curves for Sobolev functions, J. Math. Anal. Appl. 281 (2003), 171-185.

[12] C. Mariconda and G. Treu, A comparison principle and the Lipschitz continuity for minimizers, J. Convex Anal. 12 (2005) 197-212.

[13] C. Mariconda and G. Treu, Lipschitz regularity for minima without strict convexity of the Lagrangian, J. Differential Equations 243 (2007) 388-413. 
[14] C. Mariconda and G. Treu, Local Lipschitz regularity of minima for a scalar problem of the calculus of variations, Commun. Contemp. Math. 10 (2008) 1129-1149.

[15] C. Mariconda and G. Treu, Hölder regularity for a classical problem of the Calculus of Variations, Adv. Calc. Var. 2 (2009) 311-320.

Dipartimento di Matematica Pura ed Applicata, Università degli Studi di Padova, via Trieste 63, I-35121 Padova Italy

E-mail address: maricond@math.unipd.it 